Mathematical Modelling and Analysis

Volume 19 Number 2, April 2014, 241-256

http://dx.doi.org/10.3846/13926292.2014.910278

(c) Vilnius Gediminas Technical University, 2014
Publisher: Taylor\&Francis and VGTU

http://www.tandfonline.com/TMMA

Print ISSN: 1392-6292

Online ISSN: 1648-3510

\title{
An Inverse Boundary Value Problem for a Second Order Elliptic Equation in a Rectangle
}

\section{Yashar T. Mehraliyev ${ }^{a}$ and Fatma Kanca ${ }^{b}$}

${ }^{a}$ Baku State University

23, Z.I.Khalilov str., 370148 Baku, Azerbaijan

${ }^{b}$ Department of Management Information Systems, Kadir Has University

34083 Istanbul, Turkey

E-mail: yashar_aze@mail.ru

E-mail(corresp.): fatma.kanca@khas.edu.tr

Received September 7, 2012; revised February 21, 2014; published online April 15, 2014

\begin{abstract}
In this paper, the inverse problem of finding a coefficient in a second order elliptic equation is investigated. The conditions for the existence and uniqueness of the classical solution of the problem under consideration are established. Numerical tests using the finite-difference scheme combined with an iteration method is presented and the sensitivity of this scheme with respect to noisy overdetermination data is illustrated.
\end{abstract}

Keywords: inverse problem, integral overdetermination condition, elliptic equation.

AMS Subject Classification: 35J25; 35R30.

\section{Introduction}

Consider the equation

$$
u_{t t}(x, t)+u_{x x}(x, t)=a(t) u(x, t)+f(x, t)
$$

in the interior the domain $D_{T}=\{(x, t): 0 \leq x \leq 1: 0 \leq t \leq T\}$ with the boundary conditions:

$$
\begin{aligned}
& u(x, 0)=\varphi(x), \quad u_{t}(x, T)=\psi(x), \quad 0 \leq x \leq 1, \\
& u(0, t)=u(1, t), \quad u_{x}(1, t)=0, \quad 0 \leq t \leq T
\end{aligned}
$$

and the overdertermination condition

$$
\int_{0}^{1} u(x, t) d x=h(t), \quad 0 \leq t \leq T,
$$

where $f(x, t), \varphi(x), \psi(x)$ and $h(t)$ are the given functions, $u(x, t)$ and $a(t)$ are the unknown functions. 
Definition 1. We call the pair $\{u(x, t), a(t)\}$ the classical solution of inverse boundary value problem (1.1)-(1.4), if the following conditions are satisfied:

1) The function $u(x, t)$ is continuous in $D_{T}$ together with all its derivatives contained in the equation (1.1);

2) the function $a(t)$ is continuous on $[0, T]$;

3) problem (1.1)-(1.4) is satisfied in the ordinary sense.

The problem of identifying a coefficient in an elliptic equation is an interesting problem for many scientists. For surveys on the subject, we refer the reader to $[5,7,11,18]$ and the references therein.

Nonlocal problems are widely for mathematical modeling of various process of physics, chemistry, ecology and industry. For example in [2], the authors considered a nonlocal elliptic problem appearing in the theory of plasma. Nonclassical boundary and initial-boundary value problems with integral and discrete nonlocal boundary conditions were studied for various equations.(see $[2,13,15]$ and reference there in)

Various inverse problems for partial differential equations with nonlocal boundary conditions were studied in $[9,15]$

In [18], the elliptic problem for the equation

$$
-\Delta u=c(x) u+f(x) h(x, y)+g(x, y)
$$

in the rectangle is considered. Two inverse problems of finding the coefficient $c(x)$ and the right-hand side $f(x)$ from local boundary conditions are investigated in that paper. Existence and uniqueness conditions are derived for the inverse problems. The study is performed in the class of continuously differentiable functions whose derivatives satisfy a Hölder condition.

In [7], the authors investigate the convergence rates for total variation regularization of the problem of identifying (i) the coefficient $q$ in the Neumann problem for the elliptic equation $-\operatorname{div}(q \nabla u)=f$, and (ii) the coefficient $a$ in the Neumann problem for the elliptic equation $-\Delta u+a u=f$. They regularize these problems by correspondingly minimizing the convex functionals.

In [15], the inverse coefficient problem of the equation

$$
u_{x x}+\operatorname{sign}(y) u_{y y}-b^{2} u=f(x)
$$

with nonlocal boundary condition is considered. Some necessary and sufficient conditions for the uniqueness of solution of the inverse problem are found.

In [11], for the elliptic equation

$$
-\nabla(p(x) \nabla v)+\lambda q(x) v=f, \quad x \in \Omega \subset R^{n},
$$

the problem of determining when one or more of the coefficient functions $p$, $q$, and $f$ are defined uniquely by a knowledge of one or more of the solution functions $v=v_{p, q, f, \lambda}$ is considered.

In the present paper, inverse coefficient problem for elliptic equation is considered. This problem has nonlocal boundary and integral overdetermination 
condition. It should be pointed out that papers, when nonlocal inverse elliptic problems are considered mainly deal with local additional condition. Here in this paper the problem has integral overdetermination condition.

The paper is organized as follows: In Section 2, the existence and uniqueness of the solution of inverse problem (1.1)-(1.4) is proved by using the Fourier method. In Section 3, the numerical procedure for the solution of the inverse problem using the finite-difference scheme combined with an iteration method is given. Finally, in Section 4, numerical experiments are presented and discussed.

\section{Existence and Uniqueness of the Solution of the Inverse Problem}

For investigating problem (1.1)-(1.4), firstly we consider the following problem:

$$
\begin{aligned}
& y^{\prime \prime}(t)=a(t) y(t), \quad 0 \leq t \leq T, \\
& y(0)=0, \quad y^{\prime}(T)=0,
\end{aligned}
$$

where $a(t) \in C[0, T]$ is the given function, $y=y(t)$ is the unknown function, and if $y(t)$ is the solution of problem (2.1)-(2.2) then $y(t)$ is continuous on $[0, T]$ together with all its derivatives contained in equation (2.1) and satisfying conditions (2.1)-(2.2) in the ordinary sense.

The following lemma is proved.

Lemma 1. Let $\|a(t)\|_{C[0, T]} \leq R$ and

$$
\frac{1}{2} T^{2} R<1
$$

where $R$ is a constant. Then problem (2.1)-(2.2) has only a trivial solution.

Proof. It is easy to see that the homogeneous problem associated to (2.1) has only a trivial solution. Then it is known [12] that the homogeneous problem has one Green's function and boundary value problem (2.1)-(2.2) is equivalent to the integral equation

$$
y(t)=\int_{0}^{T} G(t, \tau) a(\tau) y(\tau) d \tau, \quad 0 \leq t \leq T,
$$

where

$$
G(t, \tau)= \begin{cases}-t, & t \in[0, \tau] \\ -\tau, & t \in[\tau, T] .\end{cases}
$$

Having denoted $A y(t)=\int_{0}^{T} G(t, \tau) a(\tau) y(\tau) d \tau$, we write (2.4) in the form

$$
y(t)=A y(t)
$$

and $A: C[0, T] \rightarrow C[0, T]$. 
It is easy to see that the operator $A$ is continuous in the space $C[0, T]$. Let us show that $A$ is a contraction mapping in $C[0, T]$. Indeed, for any $y(t)$, $\bar{y}(t) \in C[0, T]$ we have:

$$
\|A(y(t))-A(\bar{y}(t))\|_{C[0, T]} \leq \frac{1}{2}\|a(t)\|_{C[0, T]} T^{2}\|y(t)-\bar{y}(t)\|_{C[0, T]} .
$$

Then, using (2.3) in (2.6) we obtain $A$ is a contraction mapping in $C[0, T]$. Therefore, equation (2.5) has a unique solution $y(t) \in C[0, T]$, by Banach fixed point theorem. Thus, integral equation (2.4) has a unique solution in $C[0, T]$ and consequently, boundary value problem $(2.1)-(2.2)$ also has a unique solution in $C[0, T]$. Since $y(t)=0$ is the solution of boundary value problem $(2.1)-(2.2)$, then it has only trivial solution.

Consider the following equation:

$$
h^{\prime \prime}(t)-u_{x}(0, t)=a(t) h(t)+\int_{0}^{1} f(x, t) d x, \quad 0 \leq t \leq T .
$$

The following lemma is valid.

Lemma 2. Let $\varphi(x), \psi(x) \in C[0,1], h(t) \in C^{2}[0, T], h(t) \neq 0$ for $t \in[0, T]$, $f(x, t) \in C\left(D_{T}\right)$ and the consistency conditions

$$
\int_{0}^{1} \varphi(x) d x=h(0), \quad \int_{0}^{1} \psi(x) d x=h^{\prime}(T)
$$

be satisfied. Then the following statements are valid:

1. Each classical solution $\{u(x, t), a(t)\}$ of problem $(1.1)-(1.4)$ is the solution of problem (1.1)-(1.3), (2.7) as well;

2. each solution $\{u(x, t), a(t)\}$ of problem (1.1)-(1.3), (2.7) is a classical solution of the problem (1.1)-(1.4), if

$$
\frac{1}{2} T^{2}\|a(t)\|_{C[0, T]}<1 .
$$

Proof. Let $\{u(x, t), a(t)\}$ be a solution of problem (1.1)-(1.4). It is seen from (1.4) that

$$
\int_{0}^{1} u_{t}(x, t) d x=h^{\prime}(t), \quad \int_{0}^{1} u_{t t}(x, t) d x=h^{\prime \prime}(t), \quad 0 \leq t \leq T .
$$

Further, using (1.3) in (1.1) we have:

$$
\frac{d^{2}}{d t^{2}} \int_{0}^{1} u(x, t) d x-u_{x}(0, t)=a(t) \int_{0}^{1} u(x, t) d x+\int_{0}^{1} f(x, t) d x, 0 \leq t \leq T .
$$

Hence, from (1.4) and (2.10), (2.7) is obtained. 
Now, suppose that $\{u(x, t), a(t)\}$ is a solution of problem (1.1)-(1.3), (2.7) and (2.9) is satisfied. Then, from (2.7) and (2.11) we get:

$$
\frac{d^{2}}{d t^{2}}\left(\int_{0}^{1} u(x, t) d x-h(t)\right)=a(t)\left(\int_{0}^{1} u(x, t) d x-h(t)\right), \quad 0 \leq t \leq T .
$$

From (1.2) and (2.8) we have:

$$
\left\{\begin{array}{l}
\int_{0}^{1} u(x, 0) d x-h(0)=\int_{0}^{1} \varphi(x) d x-h(0)=0, \\
\int_{0}^{1} u_{t}(x, T) d x-h^{\prime}(T)=\int_{0}^{1} \psi(x) d x-h^{\prime}(T)=0 .
\end{array}\right.
$$

From (2.12), (2.13) and Lemma 1 the condition (1.4) is obtained.

It is known that the sequences of the functions

$$
\begin{aligned}
& X_{0}(x)=2, \quad X_{2 k-1}(x)=4 \cos \lambda_{k} x, \quad X_{2 k}(x)=4(1-x) \sin \lambda_{k} x, \\
& Y_{0}(x)=x, \quad Y_{2 k-1}(x)=x \cos \lambda_{k} x, \quad Y_{2 k}(x)=\sin \lambda_{k} x, \quad k=1,2, \ldots
\end{aligned}
$$

form in $L_{2}(0,1)$ a biorthogonal system [6], and system (2.14) forms a basis in $L_{2}(0,1)$, where $\lambda_{k}=2 \pi k(k=1,2, \ldots)$ [8]. They are also Riesz bases in $L_{2}(0,1)$ (see [9]). Then any function $g(x) \in L_{2}(0,1)$ is expanded in biorthogonal series

$$
g(x)=\sum_{k=0}^{\infty} g_{k} X_{k}(x)
$$

where $g_{k}=\left(g(x), Y_{k}(x)\right), k=0,1, \ldots$, here $($,$) is the scalar product in L_{2}(0,1)$. The estimates

$$
\frac{1}{2}\|g(x)\|_{L_{2}(0,1)}^{2} \leq \sum_{k=0}^{\infty} g_{k}^{2} \leq\|g(x)\|_{L_{2}(0,1)}^{2}
$$

are valid for any function $g(x)$ in $L_{2}(0,1)[6,8]$.

Under the suppositions $g(x) \in C^{2 i-1}[0,1], g^{(2 \mathbf{i})}(x) \in L_{2}(0,1)$ and $g^{(2 s)}(0)=g^{(2 s)}(1), g^{(2 s+1)}(1)=0(s=\overline{0, i-1} ; i \geq 1)$ the estimations are valid:

$$
\begin{aligned}
\sum_{k=1}^{\infty}\left(\lambda_{k}^{2 i} g_{2 k}\right)^{2} & \leq \frac{1}{2}\left\|g^{(2 i)}(x)\right\|_{L_{2}(0,1)}^{2} \\
\sum_{k=1}^{\infty}\left(\lambda_{k}^{2 i} g_{2 k-1}\right)^{2} & \leq \frac{1}{2}\left\|g^{(2 i)}(x) x+2 i g^{(2 i-1)}(x)\right\|_{L_{2}(0,1)}^{2}
\end{aligned}
$$

where

$$
\sum_{k=1}^{\infty}\left(\lambda_{k}^{2 i} g_{2 k}\right)^{2}=\sum_{k=1}^{\infty}\left(\lambda_{k}^{2 i} \int_{0}^{1} g(x) Y_{k}(x) d x\right)^{2}=\sum_{k=1}^{\infty}\left(\lambda_{k}^{2 i} \int_{0}^{1} g(x) \sin \lambda_{k} x d x\right)^{2},
$$

if we use integration by parts $(2 i)$ times and we use Cauchy inequalities we obtain (2.15). Similarly the other estimation is obtained. 
Under the suppositions $g(x) \in C[0,1]$ and $g^{\prime}(x) \in L_{2}(0,1)$ the estimations are valid:

$$
\sum_{k=1}^{\infty}\left(\lambda_{k} g_{2 k}\right)^{2} \leq \frac{1}{2}\left\|g^{\prime}(x)\right\|_{L_{2}(0,1)}^{2}, \sum_{k=1}^{\infty}\left(\lambda_{k} g_{2 k-1}\right)^{2} \leq \frac{1}{2}\left\|g^{\prime}(x) x+g(x)\right\|_{L_{2}(0,1)}^{2} .
$$

Under the suppositions $g(x) \in C^{2 i}[0,1], g^{(2 i+1)}(x) \in L_{2}(0,1), g^{(2 s)}(0)=$ $g^{(2 s)}(1), g^{(2 s+1)}(1)=0(i \geq 1 ; s=\overline{0, i})$ the estimations are valid:

$$
\begin{aligned}
\sum_{k=1}^{\infty}\left(\lambda_{k}^{2 i+1} g_{2 k}\right)^{2} & \leq \frac{1}{2}\left\|g^{(2 i+1)}(x)\right\|_{L_{2}(0,1)}^{2}, \\
\sum_{k=1}^{\infty}\left(\lambda_{k}^{2 i+1} g_{2 k-1}\right)^{2} & \leq \frac{1}{2}\left\|g^{(2 i+1)}(x) x+(2 i+1) g^{(2 i)}(x)\right\|_{L_{2}(0,1)}^{2} .
\end{aligned}
$$

For investigating problem (1.1)-(1.3), (2.7), consider the following spaces: The space $B_{3, T}^{2}$ [10] can be described as consisting of all functions $u(x, t)$ of the form

$$
u(x, t)=\sum_{k=1}^{\infty} u_{k}(t) X_{k}(x)
$$

considered on $D_{T}$, with the norm $\|u(x, t)\|_{B_{3, T}^{2}}=J_{T}(u)$, where

$$
\begin{aligned}
J_{T}(u) \equiv & \left\|u_{o}(t)\right\|_{C[0, T]}+\left(\sum_{k=1}^{\infty}\left(\lambda_{k}^{3}\left\|u_{2 k-1}(t)\right\|_{C[0, T]}\right)^{2}\right)^{1 / 2} \\
& +\left(\sum_{k=1}^{\infty}\left(\lambda_{k}^{3}\left\|u_{2 k}(t)\right\|_{C[0, T]}\right)^{2}\right)^{1 / 2}
\end{aligned}
$$

The space $E_{T}^{3}$ can be described as consisting of vector functions $\{u(x, t), a(t)\}$ such that $u(x, t) \in B_{3, T}^{2}, a(t) \in C[0, T]$ with the norm

$$
\|z\|_{E_{T}^{3}}=\|u(x, t)\|_{B_{3, T}^{2}}+\|a(t)\|_{C[0, T]} .
$$

It is obvious that $E_{T}^{3}$ and $B_{3, T}^{2}$ are Banach spaces. The solution of the problem (1.1)-(1.3) can be written in the form:

$$
u(x, t)=\sum_{k=1}^{\infty} u_{k}(t) X_{k}(x)
$$

where $u_{k}(t)=\int_{0}^{1} u(x, t) Y_{k}(x) d x$ is a solution of the following system:

$$
\begin{aligned}
& u_{0}^{\prime \prime}(t)=F_{0}(t ; u, a), \quad 0 \leq t \leq T, \\
& u_{2 k}^{\prime \prime}(t)-\lambda_{k}^{2} u_{2 k}(t)=F_{2 k}(t ; u, a), \quad 0 \leq t \leq T ; \quad k=1,2, \ldots, \\
& u_{2 k-1}^{\prime \prime}(t)-\lambda_{k}^{2} u_{2 k-1}(t)=F_{2 k-1}(t ; u, a)+2 \lambda_{k} u_{2 k}(t), \quad 0 \leq t \leq T ; k=1,2, \ldots, \\
& u_{k}(0)=\varphi_{k}, u_{k}^{\prime}(T)=\psi_{k}, \quad k=0,1, \ldots,
\end{aligned}
$$


where

$$
\begin{aligned}
& F_{k}(t ; u, a)=a(t) u_{k}(t)+f_{k}(t), \quad f_{k}(t)=\int_{0}^{1} f(x, t) Y_{k}(x) d x \\
& \varphi_{k}=\int_{0}^{1} \varphi(x) Y_{k}(x) d x, \quad \psi_{k}=\int_{0}^{1} \psi(x) Y_{k}(x) d x, \quad k=0,1, \ldots
\end{aligned}
$$

Solving the problem (2.20) we find:

$$
\begin{aligned}
& u_{0}(t)=\varphi_{0}+\psi_{0} t+\int_{0}^{T} G_{0}(t, \tau) F_{0}(\tau ; u, a) d \tau, \\
& u_{2 k}(t)=\frac{\cosh \left(\lambda_{k}(T-t)\right)}{\cosh \left(\lambda_{k} T\right)} \varphi_{2 k}+\frac{\sinh \left(\lambda_{k} t\right)}{\lambda_{k} \cosh \left(\lambda_{k} T\right)} \psi_{2 k}+\int_{0}^{T} G_{k}(t, \tau) F_{2 k}(\tau ; u, a) d \tau, \\
& u_{2 k-1}(t)=\frac{\cosh \left(\lambda_{k}(T-t)\right)}{\cosh \left(\lambda_{k} T\right)} \varphi_{2 k-1}+\frac{\sinh \left(\lambda_{k} t\right)}{\lambda_{k} \cosh \left(\lambda_{k} T\right)} \psi_{2 k-1} \\
& \quad+\int_{0}^{T} G_{k}(t, \tau) F_{2 k-1}(\tau ; u, a) d \tau+\frac{1}{\cosh ^{2}\left(\lambda_{k} T\right)} \\
& \quad \times\left\{\left[T \operatorname{sh}\left(\lambda_{k} t\right)+t \operatorname{ch}\left(\lambda_{k} T\right) \operatorname{sh}\left(\lambda_{k}(T-t)\right)\right] \varphi_{2 k}+\left[-T \sinh \left(\lambda_{k} T\right) \sinh \left(\lambda_{k} t\right)\right.\right. \\
& \left.\left.\quad+t \cosh \left(\lambda_{k} T\right) \cosh \left(\lambda_{k} t\right)-\frac{1}{\lambda_{k}} \cosh \left(\lambda_{k} T\right) \sinh \left(\lambda_{k} t\right)\right] \frac{1}{\lambda_{k}} \psi_{2 k}\right\} \\
& \quad+\int_{0}^{T} G_{k}(t, \tau)\left(\int_{0}^{T} G_{k}(t, \xi) F_{2 k}(\xi ; u, a) d \xi\right) d \tau,
\end{aligned}
$$

where

$$
\begin{aligned}
& G_{0}(t, \tau)=\left\{\begin{array}{ll}
-t, & t \in[0, \tau], \\
-\tau, & t \in[\tau, T],
\end{array} G_{k}(t, \tau)= \begin{cases}g_{1 k}(t, \tau), & t \in[0, \tau], \\
g_{2 k}(t, \tau), & t \in[\tau, T],\end{cases} \right. \\
& g_{1 k}(t, \tau)=\frac{-1}{2 \lambda_{k} \cosh \left(\lambda_{k} T\right)}\left[\sinh \left(\lambda_{k}(T+t-\tau)\right)-\sinh \left(\lambda_{k}(T-(t+\tau))\right)\right], \\
& g_{2 k}(t, \tau)=\frac{1}{2 \lambda_{k} \cosh \left(\lambda_{k} T\right)}\left[\sinh \left(\lambda_{k}(T-(t+\tau))\right)-\sinh \left(\lambda_{k}(T-(t-\tau))\right)\right] .
\end{aligned}
$$

After substituting (2.21) into (2.19), we get:

$$
\begin{aligned}
u(x, t)= & \left(\varphi_{0}+\psi_{0} t+\int_{0}^{T} G_{0}(t, \tau) F_{0}(\tau ; u, a) d \tau\right) X_{0}(x) \\
& +\sum_{k=1}^{\infty}\left\{\frac{\cosh \left(\lambda_{k}(T-t)\right)}{\cosh \left(\lambda_{k} T\right)} \varphi_{2 k}+\frac{\sinh \left(\lambda_{k} t\right)}{\lambda_{k} \cosh \left(\lambda_{k} T\right)} \psi_{2 k}\right. \\
& \left.+\int_{0}^{T} G_{k}(t, \tau) F_{2 k}(\tau ; u, a) d \tau\right\} X_{2 k}(x) \\
& +\sum_{k=1}^{\infty}\left\{\frac{\cosh \left(\lambda_{k}(T-t)\right)}{\cosh \left(\lambda_{k} T\right)} \varphi_{2 k-1}+\frac{\sinh \left(\lambda_{k} t\right)}{\lambda_{k} \cosh \left(\lambda_{k} T\right)} \psi_{2 k-1}\right.
\end{aligned}
$$




$$
\begin{aligned}
& +\int_{0}^{T} G_{k}(t, \tau) F_{2 k-1}(\tau ; u, a) d \tau \\
& +\frac{1}{\cosh ^{2}\left(\lambda_{k} T\right)}\left\{\left[T \sinh \left(\lambda_{k} t\right)+t \cosh \left(\lambda_{k} T\right) \sinh \left(\lambda_{k}(T-t)\right)\right] \varphi_{2 k}\right. \\
& +\left[-T \sinh \left(\lambda_{k} T\right) \sinh \left(\lambda_{k} t\right)+t \cosh \left(\lambda_{k} T\right) \cosh \left(\lambda_{k} t\right)\right. \\
& \left.\left.-\frac{1}{\lambda_{k}} \cosh \left(\lambda_{k} T\right) \sinh \left(\lambda_{k} t\right)\right] \frac{1}{\lambda_{k}} \psi_{2 k}\right\} \\
& \left.+\int_{0}^{T} G_{k}(t, \tau)\left(\int_{0}^{T} G_{k}(t, \xi) F_{2 k}(\xi ; u, a) d \xi\right) d \tau\right\} X_{2 k-1}(x)
\end{aligned}
$$

From (2.7), (2.19) and (2.21) we obtain:

$$
\begin{aligned}
a(t)= & h^{-1}(t)\left\{h^{\prime \prime}(t)-\int_{0}^{1} f(x, t) d x-4 \sum_{k=1}^{\infty} \lambda_{k}\left[\frac{\cosh \left(\lambda_{k}(T-t)\right)}{\cosh \left(\lambda_{k} T\right)} \varphi_{2 k}\right.\right. \\
& \left.\left.+\frac{\sinh \left(\lambda_{k} t\right)}{\lambda_{k} \cosh \left(\lambda_{k} T\right)} \psi_{2 k}+\int_{0}^{T} G_{k}(t, \tau) F_{2 k}(\tau ; u, a) d \tau\right]\right\} .
\end{aligned}
$$

Thus the solution of problem $(1.1)-(1.3),(2.7)$ is reduced to the solution of system (2.22), (2.23).

Using the definition of the solution of the problem (1.1)-(1.3), (2.7), similar to $[16]$ we prove the following lemma.

Lemma 3. If $\{u(x, t), a(t)\}$ is any solution of problem $(1.1)-(1.3),(2.7)$, then the functions

$$
u_{k}(t)=\int_{0}^{1} u(x, t) Y_{k}(x) d x, \quad k=0,1, \ldots
$$

satisfy system $(2.21)$ on $[0, T]$.

Now, in the space $E_{T}^{3}$ consider the operator $\Phi(u, a)=\left\{\Phi_{1}(u, a), \Phi_{2}(u, a)\right\}$, where

$$
\Phi_{1}(u, a)=\widetilde{u}(x, t) \equiv \sum_{k=1}^{\infty} \widetilde{u}_{k}(t) X_{k}(x), \quad \Phi_{2}(u, a)=\widetilde{a}(t)
$$

and $\widetilde{u}_{0}(t), \widetilde{u}_{2 k}(t), \widetilde{u}_{2 k-1}(t), k=1,2, \ldots$, and $\widetilde{a}(t)$ are equal to the right hand sides of (2.21) and (2.23), respectively. It is easy to see that

$$
\begin{aligned}
& \frac{\sinh \left(\lambda_{k} t\right)}{\cosh \left(\lambda_{k} T\right)} \leq 1, \quad 0 \leq t \leq T, \quad \frac{\cosh \left(\lambda_{k} t\right)}{\cosh \left(\lambda_{k} T\right)} \leq 2, \quad 0 \leq t \leq T, \\
& \frac{\sinh \left(\lambda_{k}(T-t)\right)}{\left.\cosh \lambda_{k} T\right)} \leq 2, \quad 0 \leq t \leq T, \quad \frac{\sinh \left(\lambda_{k}(T+t-\tau)\right)}{\cosh \left(\lambda_{k} T\right)} \leq 1, \quad 0 \leq t \leq \tau \leq T, \\
& \frac{\sinh \left(\lambda_{k}(T-(t+\tau))\right)}{\cosh \left(\lambda_{k} T\right)} \leq 1, \quad 0 \leq t \leq \tau \leq T, \\
& \frac{\sinh \left(\lambda_{k}(T-(t+\tau))\right)}{\cosh \left(\lambda_{k} T\right)} \leq 1, \quad \frac{\sinh \left(\lambda_{k}(T-(t-\tau))\right)}{\cosh \left(\lambda_{k} T\right)} \leq 1, \quad 0 \leq \tau \leq t \leq T .
\end{aligned}
$$


Taking into account these relations, with the help of simple transformations we find:

$$
\begin{aligned}
& \left\|\widetilde{u}_{0}(t)\right\|_{C[0, T]} \leq\left|\varphi_{0}\right|+T\left|\psi_{0}\right|+2 T \sqrt{T}\left(\int_{0}^{T}\left|f_{o}(\tau)\right|^{2} d \tau\right)^{1 / 2} \\
& +2 T^{2}\|a(t)\|_{C[0, T]}\left\|u_{0}(t)\right\|_{C[0, T]}, \\
& \left(\sum_{k=1}^{\infty}\left(\lambda_{k}^{3}\left\|\widetilde{u}_{2 k}(t)\right\|_{C[0, T]}\right)^{2}\right)^{1 / 2} \leq 4\left(\sum_{k=1}^{\infty}\left(\lambda_{k}^{3}\left|\varphi_{2 k}\right|\right)^{2}\right)^{1 / 2}+2\left(\sum_{k=1}^{\infty}\left(\lambda_{k}^{2}\left|\psi_{2 k}\right|\right)^{2}\right)^{1 / 2} \\
& +2 \sqrt{T}\left(\int_{0}^{T} \sum_{k=1}^{\infty}\left(\lambda_{k}^{2}\left|f_{2 k}(\tau)\right|\right)^{2} d \tau\right)^{1 / 2} \\
& +2 T\|a(t)\|_{C[0, T]}\left(\sum_{k=1}^{\infty}\left(\lambda_{k}^{2}\left\|u_{2 k}(t)\right\|_{C[0, T]}\right)^{2}\right)^{1 / 2}, \\
& \left(\sum_{k=1}^{\infty}\left(\lambda_{k}^{3}\left\|\widetilde{u}_{2 k-1}(t)\right\|_{C[0, T]}\right)^{2}\right)^{1 / 2} \leq 4 \sqrt{2}\left(\sum_{k=1}^{\infty}\left(\lambda_{k}^{3}\left|\varphi_{2 k-1}\right|\right)^{2}\right)^{1 / 2} \\
& +2 \sqrt{2}\left(\sum_{k=1}^{\infty}\left(\lambda_{k}^{2}\left|\psi_{2 k-1}\right|\right)^{2}\right)^{1 / 2}+2 \sqrt{2 T}\left(\int_{0}^{T} \sum_{k=1}^{\infty}\left(\lambda_{k}^{2}\left|f_{2 k-1}(\tau)\right|\right)^{2} d \tau\right)^{1 / 2} \\
& +2 \sqrt{2} T\|a(t)\|_{C[0, T]}\left(\sum_{k=1}^{\infty}\left(\lambda_{k}^{2}\left\|u_{2 k-1}(t)\right\|_{C[0, T]}\right)^{2}\right)^{1 / 2} \\
& +4 T\left(\sum_{k=1}^{\infty}\left(\lambda_{k}^{3}\left|\varphi_{2 k}\right|\right)^{2}\right)^{1 / 2}+2 \sqrt{2}(1+3 T)\left(\sum_{k=1}^{\infty}\left(\lambda_{k}^{2}\left|\psi_{2 k}\right|\right)^{2}\right)^{1 / 2} \\
& +4 \sqrt{2} T \sqrt{T}\left(\int_{0}^{T} \sum_{k=1}^{\infty}\left(\lambda_{k}\left|f_{2 k}(\tau)\right|\right)^{2} d \tau\right)^{1 / 2} \\
& +4 \sqrt{2} T^{2}\|a(t)\|_{C[0, T]}\left(\sum_{k=1}^{\infty}\left(\lambda_{k}\left\|u_{2 k}(t)\right\|_{C[0, T]}\right)^{2}\right)^{1 / 2}, \\
& \|\widetilde{a}(t)\|_{C[0, T]} \leq\left\|h^{-1}(t)\right\|_{C[0, T]}\left\{\left\|h^{\prime \prime}(t)-\int_{0}^{1} f(x, t) d x\right\|_{C[0, T]}\right. \\
& +\frac{4}{\sqrt{6}}\left(\sum_{k=1}^{\infty}\left(\lambda_{k}^{2}\left|\varphi_{2 k}\right|\right)^{2}\right)^{1 / 2}+\frac{2}{\sqrt{6}}\left(\sum_{k=1}^{\infty}\left(\lambda_{k}\left|\psi_{2 k}\right|\right)^{2}\right)^{1 / 2} \\
& +\frac{2}{\sqrt{6}} \sqrt{T}\left(\int_{0}^{T} \sum_{k=1}^{\infty}\left(\lambda_{k}\left|f_{2 k}(\tau)\right|\right)^{2} d \tau\right)^{1 / 2} \\
& \left.+\frac{2}{\sqrt{6}} T\|a(t)\|_{C[0, T]}\left(\sum_{k=1}^{\infty}\left(\lambda_{k}\left\|u_{2 k}(t)\right\|_{C[0, T]}\right)^{2}\right)\right\} .
\end{aligned}
$$

Suppose that the data of problem (1.1)-(1.3), (2.7) satisfy the following conditions: 
$\left(\mathrm{C}_{1}\right) \varphi(x) \in C^{2}[0,1], \varphi^{\prime \prime \prime}(x) \in L_{2}(0,1), \varphi(0)=\varphi(1), \varphi^{\prime}(1)=0, \varphi^{\prime \prime}(0)=\varphi^{\prime \prime}(1) ;$

$\left(\mathrm{C}_{2}\right) \psi(x) \in C^{1}[0,1], \psi^{\prime \prime}(x) \in L_{2}(0,1), \psi(0)=\psi(1), \psi^{\prime}(1)=0 ;$

$\left(\mathrm{C}_{3}\right) f(x, t), f_{x}(x, t) \in C\left(D_{T}\right) ; f_{x x}(x, t) \in L_{2}\left(D_{T}\right), f(0, t)=f(1, t), f_{x}(1, t)=0$, $0 \leq t \leq T$

$\left(\mathrm{C}_{4}\right) h(t) \in C^{2}[0, T], h(t) \neq 0, \forall t \in[0, T]$.

Then, considering (2.15)-(2.18) in (2.24)-(2.27) we get:

$$
\begin{aligned}
\|\widetilde{u}(x, t)\|_{B_{3, T}^{2}} & \leq A_{1}(T)+B_{1}(T)\|a(t)\|_{C[0, T]}\|u(x, t)\|_{B_{3, T}^{2}}, \\
\|\widetilde{a}(t)\|_{C[0, T]} & \leq A_{2}(T)+B_{2}(T)\|a(t)\|_{C[0, T]}\|u(x, t)\|_{B_{2, T}^{3}},
\end{aligned}
$$

where

$$
\begin{aligned}
A_{1}(T)= & \|\varphi(x) x\|_{L_{2}(0,1)}+T\|\psi(x) x\|_{L_{2}(0,1)}+2 T \sqrt{T}\|f(x, t)\|_{L_{2}\left(D_{T}\right)} \\
& +2 \sqrt{2}(1+T)\left\|\varphi^{\prime \prime \prime}(x)\right\|_{L_{2}(0,1)}+\sqrt{2}(1+\sqrt{2}+3 \sqrt{2} T)\left\|\psi^{\prime \prime}(x)\right\|_{L_{2}(0,1)} \\
& +\sqrt{2 T}(1+2 \sqrt{2} T)\left\|f_{x x}(x, t)\right\|_{L_{2}\left(D_{T}\right)}+4\left\|\varphi^{\prime \prime \prime}(x) x+3 \varphi^{\prime \prime}(x)\right\|_{L_{2}(0,1)} \\
& +2\left\|\psi^{\prime \prime}(x) x+2 \psi^{\prime}(x)\right\|_{L_{2}(0,1)}+2 \sqrt{T}\left\|f_{x x}(x, t) x+2 f_{x}(x, t)\right\|_{L_{2}\left(D_{T}\right)}, \\
B_{1}(T)= & 2(1+\sqrt{2}) T+2(1+2 \sqrt{2}) T^{2}, \\
A_{2}(T)= & \left\|h^{-1}(t)\right\|_{C[0, T]}\left\{\left\|h^{\prime \prime}(t)-\int_{0}^{1} f(x, t) d x\right\|_{C[0, T]}+\frac{2 \sqrt{3}}{3}\left\|\varphi^{\prime \prime}(x)\right\|_{L_{2}(0,1)}\right. \\
& \left.+\frac{\sqrt{3}}{3}\left\|\psi^{\prime}(x)\right\|_{L_{2}(0,1)}+\frac{\sqrt{3 T}}{3}\left\|f_{x}(x, t)\right\|_{L_{2}\left(D_{T}\right)}\right\}, \\
& \frac{\sqrt{3}}{3} T\left\|h^{-1}(t)\right\|_{C[0, T]} .
\end{aligned}
$$

From inequalities (2.28), (2.29) we deduce:

$$
\|\widetilde{u}(x, t)\|_{B_{2, T}^{3}}+\|\widetilde{a}(t)\|_{C[0, T]} \leq A(T)+B(T)\|a(t)\|_{C[0, T]}\|u(x, t)\|_{B_{3, T}^{2}},
$$

where $A(T)=A_{1}(T)+A_{2}(T), B(T)=B_{1}(T)+B_{2}(T)$.

So, we can prove the following theorem:

Theorem 1. Let conditions $\left(C_{1}\right)-\left(C_{4}\right)$ be satisfied, and

$$
(A(T)+2)^{2} B(T)<1 .
$$

Then problem (1.1)-(1.3), (2.7) has a unique solution in the sphere $K=$ $K_{R}\left(\|z\|_{E_{T}^{3}} \leq R=A(T)+2\right)$ of the space $E_{T}^{3}$.

Proof. In the space $E_{T}^{3}$ consider the equation

$$
z=\Phi z
$$


where $z=\{u, a\}$, the components $\Phi_{i}(u, a)(i=1,2)$ of the operator $\Phi(u, a)$ are determined by the right hand sides of equations (2.22) and (2.23).

Consider the operator $\Phi(u, a)$ in the sphere $K=K_{R}$ from $E_{T}^{3}$. Similar to $(2.30)$, we get that for any $z, z_{1}, z_{2} \in K_{R}$ the following estimations are valid:

$$
\begin{aligned}
& \|\Phi z\|_{E_{T}^{3}} \leq A(T)+B(T)\|a(t)\|_{C[0, T]}\|u(x, t)\|_{B_{2, T}^{3}} \\
& \left\|\Phi z_{1}-\Phi z_{2}\right\|_{E_{T}^{3}} \leq B(T) R\left(\left\|a_{1}(t)-a_{2}(t)\right\|_{C[0, T]}+\left\|u_{1}(x, t)-u_{2}(x, t)\right\|_{B_{3, T}^{2}}\right) .
\end{aligned}
$$

Then, using (2.31), it follows from estimations (2.33) that the operator $\Phi$ acts in the sphere $K=K_{R}$ and it is a contraction mapping. Therefore, in the sphere $K=K_{R}$, the operator $\Phi$ has a unique fixed point $\{u, a\}$ that is a solution of equation (2.32).

The function $u(x, t)$, as the element of the space $B_{3, T}^{2}$, has continuous derivatives $u(x, t), u_{x}(x, t)$ and $u_{x x}(x, t)$ in $D_{T}$. Now, from (2.20) we get:

$$
\begin{aligned}
& \left\|u_{0}^{\prime \prime}(t)\right\|_{C[0, T]} \leq\|a(t)\|_{C[0, T]}\left\|u_{0}(t)\right\|_{C[0, T]}+\|\| f(x, t)\left\|_{C[0, T]}\right\|_{L_{2}(0,1)}, \\
& \left(\sum_{k=1}^{\infty}\left(\lambda_{k}\left\|u_{2 k}^{\prime \prime}(t)\right\|_{C(0, T)}\right)^{2}\right)^{1 / 2} \leq \sqrt{2}\left(\sum_{k=1}^{\infty}\left(\lambda_{k}^{3}\left\|u_{2 k}(t)\right\|_{C(0, T)}\right)^{2}\right)^{1 / 2} \\
& \quad+\|\| a(t) u_{x}(x, t)+f_{x}(x, t)\left\|_{C[0, T]}\right\|_{L_{2}(0,1)}, \\
& \left(\sum_{k=1}^{\infty}\left(\lambda_{k}\left\|u_{2 k-1}^{\prime \prime}(t)\right\|_{C(0, T)}\right)^{2}\right)^{1 / 2} \\
& \leq \sqrt{3}\left(\sum_{k=1}^{\infty}\left(\lambda_{k}^{3}\left\|u_{2 k-1}(t)\right\|_{C(0, T)}\right)^{2}\right)^{1 / 2}+\sqrt{6}\left\|u_{x x}(x, t)\right\|_{C\left(D_{T}\right)} \\
& \quad+\frac{\sqrt{3}}{2}\|\| a(t)\left(u_{x}(x, t) x+u(x, t)\right)+f_{x}(x, t) x+f(x, t)\left\|_{C[0, T]}\right\|_{L_{2}(0,1)} .
\end{aligned}
$$

Hence it follows that $u_{t t}(x, t)$ is continuous in $D_{T}$.

It is easy to verify that equation (1.1) and conditions (1.2), (1.3) and (2.7) are satisfied in the ordinary sense. Consequently, $\{u(x, t), a(t)\}$ is a solution of problem (1.1)-(1.3), (2.7), and by Lemma 3 it is unique.

The following theorem is proved by means of Lemma 2 .

Theorem 2. Let all the conditions of Theorem 1,

$$
\frac{1}{2}(A(T)+2) T^{2}<1
$$

and consistency conditions

$$
\int_{0}^{1} \varphi(x) d x=h(0), \quad \int_{0}^{1} \psi(x) d x=h^{\prime}(T) .
$$

be satisfied. Then in the sphere $K=K_{R}\left(\|z\|_{E_{T}^{3}} \leq A(T)+2\right)$ of the space $E_{T}^{3}$, problem (1.1)-(1.4) has a unique classical solution. 
Remark 1. The condition (2.32) in Theorem 1 implies that the mapping P acting in the sphere $K_{R}$ is contraction mapping, this condition is sufficient for applying Banach fixed point theorem, Therefore, the unique solvability of problem (1.1)-(1.3), (2.7) is obtained . This problem is equivalent to the original problem (1.1)-(1.4) under similar condition in Theorem 2. To check these conditions is not easy but they are satisfied for small $T$.

Remark 2. The existence and uniqueness of the solution of inverse problem (1.1)-(1.3) are obtained in a sphere $K_{R}$ under some sufficient conditions. These conditions are also satisfied for small $T$. Such a condition is popular in the theory of inverse BVP (see $[3,4,9,14]$ ). The investigation method in $[3,4]$ allows them to continue local solution to global solution.

\section{$3 \quad$ Numerical Procedure}

We use the finite difference method with an iteration to problem (1.1)-(1.4) [17].

We subdivide the intervals $[0,1]$ and $[0, T]$ into subintervals $M$ and $N$ of equal lengths $h=\frac{1}{M}$ and $\tau=\frac{T}{N}$, respectively.

The finite difference scheme for (1.1)-(1.4) is as follows:

$$
\begin{aligned}
& \frac{u_{i}^{j+1}-2 u_{i}^{j}+u_{i}^{j-1}}{\tau^{2}}+\frac{u_{i+1}^{j}-2 u_{i}^{j}+u_{i-1}^{j}}{h^{2}}=a^{j} u_{i}^{j}+f_{i}^{j}, \\
& u_{i}^{0}=\phi_{i}, \quad u_{i}^{N}=\tau \Psi_{i}+u_{i}^{N-1}, \\
& u_{0}^{j}=u_{M}^{j}, \quad u_{M-1}^{j}=u_{M+1}^{j},
\end{aligned}
$$

where $1 \leq i \leq M$ and $0 \leq j \leq N$ are the indices for the spatial and time steps respectively, $u_{i}^{j}=u\left(x_{i}, t_{j}\right), \bar{a}_{i}^{j}=a\left(t_{j}\right), \phi_{i}=\varphi\left(x_{i}\right), \Psi_{i}=\psi\left(x_{i}\right), f_{i}^{j}=f\left(x_{i}, t_{j}\right)$, $x_{i}=i h, t_{j}=j \tau$.

From (3.1) we can write

$$
u_{i}^{j}=\frac{1}{2\left(h^{2}+\tau^{2}\right)+h^{2} \tau^{2} a^{j}}\left(h^{2}\left(u_{i}^{j+1}+u_{i}^{j-1}\right)+\tau^{2}\left(u_{i+1}^{j}+u_{i-1}^{j}\right)-h^{2} \tau^{2} f_{i}^{j}\right) .
$$

Now let us construct the iteration. First, integrating the equation (1.1) with respect to $x$ from 0 to 1 and using (1.3) and (1.4), we obtain

$$
a(t)=\frac{h^{\prime \prime}(t)-u_{x}(0, t)-\int_{0}^{1} f(x, t) d x}{h(t)} .
$$

The finite difference approximation of this equation

$$
a^{j}=\frac{\left[\left(h^{j+1}-2 h^{j}+h^{j-1}\right) / \tau^{2}\right]-\left[\left(u_{1}^{j}-u_{0}^{j}\right) / h\right]-(f i n)^{j}}{h^{j}},
$$

where $h^{j}=h\left(t_{j}\right),(f i n)^{j}=\int_{0}^{1} f\left(x, t_{j}\right) d x, j=0,1, \ldots, N$.

We denote the values of $a^{j}, u_{i}^{j}$ at the $s$-th iteration step $a^{j(s)}, u_{i}^{j(s)}$, respectively. In numerical computation, since the time step is very small, we can 
take $a^{j+1(0)}=r^{j}, u_{i}^{j+1(0)}=u_{i}^{j}, j=0,1,2, \ldots, N, i=1,2, \ldots, M$. At each $(s+1)$-th iteration step we first determine $a^{j+1(s+1)}$ from the formula

$$
a^{j+1(s+1)}=\frac{\left[\left(h^{j+2}-2 h^{j+1}+h^{j}\right) / \tau^{2}\right]-\left[\left(u_{1}^{j+1(s)}-u_{0}^{j+1(s)}\right) / h\right]-(f i n)^{j+1}}{h^{j+1}} .
$$

Then from (3.4) we determine

$$
u_{i}^{j+1(s+1)}=\frac{\left(h^{2}\left(u_{i}^{j+2(s)}+u_{i}^{j(s)}\right)+\tau^{2}\left(u_{i+1}^{j+1(s)}+u_{i-1}^{j+1(s)}\right)-h^{2} \tau^{2} f_{i}^{j}\right)}{2\left(h^{2}+\tau^{2}\right)+h^{2} \tau^{2} a^{j+1(s+1)}} .
$$

If the difference of values between two iterations reaches the prescribed tolerance, the iteration is stopped and we accept the corresponding values $a^{j+1(s+1)}, u_{i}^{j+1(s+1)}(i=1,2, \ldots, M)$ as $a^{j+1}, u_{i}^{j+1}(i=1,2, \ldots, M, j=$ $0,2, \ldots, N-1)$, respectively.

\section{Numerical Examples and Discussions}

In this section, we present two examples to illustrate the efficiency of the numerical method described in the previous section.

Example 1. Consider inverse problem (1.1)-(1.4), with

$$
\begin{aligned}
& f(x, t)=-(1+\cos (2 \pi x)) \exp (6 t)-(2 \pi)^{2}(1+\cos (2 \pi x)) \exp (t), \\
& \varphi(x)=1+\cos (2 \pi x), \quad \psi(x)=(1+\cos (2 \pi x)) \exp (T), \\
& h(t)=\exp (t), \quad x \in[0,1], t \in[0, T] .
\end{aligned}
$$

It is easy to check that the exact solution of problem (1.1)-(1.4) is

$$
\{a(t), u(x, t)\}=\{1+\exp (5 t),(1+\cos (2 \pi x)) \exp (t)\} .
$$

Let us apply the scheme of the previous section for the step sizes $h=0.01$, $\tau=h$. Figure 1 shows the exact and the numerical solutions of $\{a(t), u(x, T)\}$, when $T=1$ and the input data (1.4) is exact.

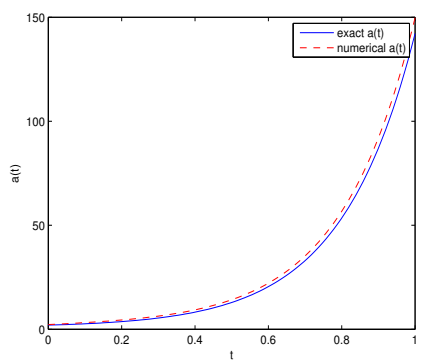

a)

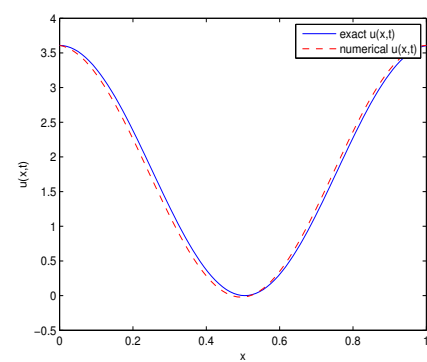

b)

Figure 1. The exact and numerical solutions for $T=1$ : a) $a(t), \mathrm{b}) u(x, T)$.

From these figures it can be seen that the agreement between the numerical and exact solutions for $a(t)$ and $u(x, T)$ is good. 
(a)

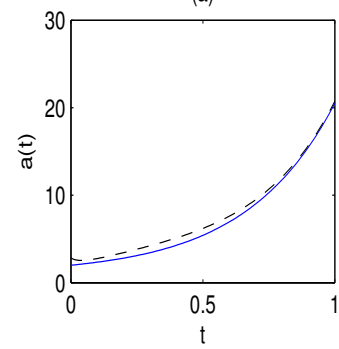

(c)

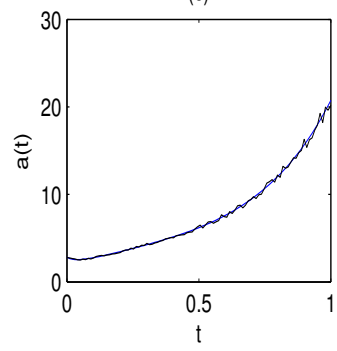

(b)

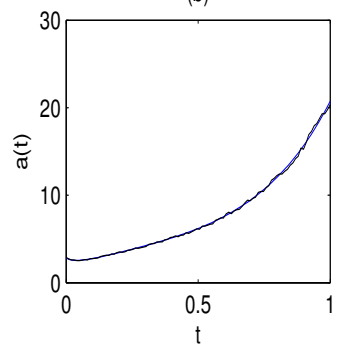

Figure 2. The numerical solutions of $a(t)$ (a) for $0 \%$ noisy data, (b) for $3 \%$ noisy data, (c) for $5 \%$ noisy data.

Example 2. Consider inverse problem (1.1)-(1.4), with

$$
\begin{aligned}
& f(x, t)=-(1-x) \sin (2 \pi x) \exp (4 t) \\
& -\left[(2 \pi)^{2}(1-x) \sin (2 \pi x)+4 \pi \cos (2 \pi x)\right] \exp (t), \\
& \varphi(x)=(1-x) \sin (2 \pi x), \quad \psi(x)=(1-x) \sin (2 \pi x) \exp (T), \\
& h(t)=\frac{1}{2 \pi} \exp (t), \quad x \in[0,1], t \in[0, T] .
\end{aligned}
$$

It is easy to check that the exact solution of problem (1.1)-(1.4) is

$$
\{a(t), u(x, t)\}=\{1+\exp (3 t),(1-x) \sin (2 \pi x) \exp (t)\} .
$$

Let us apply the scheme of the previous section for the step sizes $h=0.01$, $\tau=h$.

Next, we will illustrate the stability of the numerical solution with respect to the noisy overdetermination data (1.4), defined by the function

$$
h_{\gamma}(t)=h(t)(1+\gamma \theta) \text {, }
$$

where $\gamma$ is the percentage of noise and $\theta$ are random variables generated from a uniform distribution in the interval $[-1,1]$. Figures 2 and 3 show the exact and the numerical solution of $\{a(t), u(x, t)\}$ when the input data (1.4) is contaminated by $\gamma=3 \%$ and $5 \%$ noise.

Numerical differentiation is used to compute the values of $h^{\prime \prime}(t)$ and $u_{x}(0, t)$ in the formula $a(t)$ It is well known that numerical differentiation is slightly 
(a)

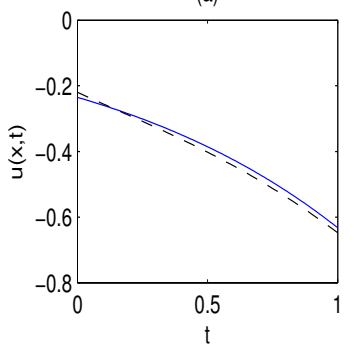

(c)

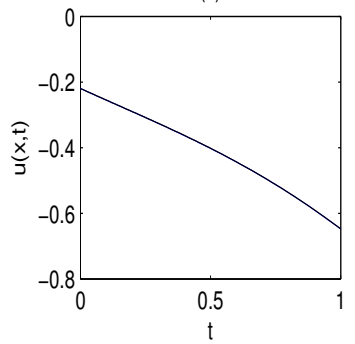

(b)

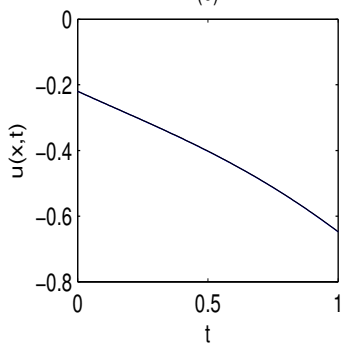

Figure 3. The numerical solutions of $u(x, T)$ for $T=1$, (a) for $0 \%$ noisy data, (b) for $3 \%$ noisy data, (c) for $5 \%$ noisy data.

ill-posed and it can cause some numerical difficulties. One can apply the cubic spline function technique [1] to get a decent accuracy. The condition number of the system grows, if the overdetermination data $h(t)$ fast decreases in $t$. Therefore it causes some numerical difficulties.

\section{Conclusion}

The inverse problem of finding a coefficient in a second order elliptic equation with nonlocal boundary and integral overdetermination conditions has been considered. This inverse problem has been investigated from both theoretical and numerical points of view. In the theoretical part of the article, the conditions for the existence and uniqueness of the problem have been established. In the numerical part, the sensitivity of finite-difference scheme combined with an iteration method with respect to noisy overdetermination data has been illustrated.

\section{Acknowledgement}

The authors are grateful to Professor M.I. Ismailov for many valuable suggestions and constructive comments that have greatly improved the presentation of this paper. 


\section{References}

[1] K.E. Atkinson. Elementary Numerical Analysis. John Wiley, New York, 1985.

[2] A.V. Bidsadze and A.A. Samarskii. On some simple generalization of linear elliptic boundary value problems. Sov. Math. Dokl., 10:398-400, 1969.

[3] J.R. Cannon and Y. Lin. Determination of parameter $p(t)$ in Hölder classes for some semilinear parabolic equations. Inverse Problems, 4:595-606, 1988. http://dx.doi.org/10.1088/0266-5611/4/3/005.

[4] J.R. Cannon, Y. Lin and S. Wang. Determination of a control parameter in a parabolic differential equation. J. Austral. Math. Soc. Ser. B, 33:149-163, 1991. http://dx.doi.org/10.1017/S0334270000006962.

[5] A.M. Denisov. Introduction to Inverse Problem Theory. Nauka, Moscow, 1994. (In Russian)

[6] I.C. Gohberg and M.G. Krein. Introduction to the Theory of Linear Nonselfadjoint Operators. Amer. Math. Soc., Providence, RI, 1969.

[7] D.N. Hao and T.N.T. Quyen. Convergence rates for total variation regularization of coefficient identification problems in elliptic equations l. Inverse Problems, 27, 2011. http://dx.doi.org/10.1088/0266-5611/27/7/075008.

[8] N.I. Ionkin. Solution of a boundary-value problem in heat conduction with a nonclassical boundary condition. Differential Equations, 13:204-211, 1977.

[9] M.I. Ismailov and F. Kanca. An inverse coefficient problem for a parabolic equation in the case of nonlocal boundary and overdetermination conditions. Math. Meth. Appl. Sci., 34(6):692-702, 2011. http://dx.doi.org/10.1002/mma.1396.

[10] K.I. Khudaverdiyev and A.G. Alieva. On the global existence of solution to one-dimensional fourth order nonlinear Sobolev type equations. Appl. Math. Comput., 217:347-354, 2010. http://dx.doi.org/10.1016/j.amc.2010.05.067.

[11] I. Knowles. Uniqueness for an elliptic inverse problem. SIAM J. Appl. Math., 59(4):1356-1370, 1999. http://dx.doi.org/10.1137/S0036139997327782.

[12] M.A. Naimark. Linear Differential Equations. Nauka, Moscow, 1969. (In Russian)

[13] Ya.A. Roitberg and Z.G. Sheftel. Nonlocal problems for elliptic equations and systems. Sib. Math. J., 13(1):165-181, 1972.

http://dx.doi.org/10.1007/BF00967646.

[14] V.G. Romanov. Inverse Problems of Mathematical Physics. VNU Science Press, 1986.

[15] K.B. Sabitov and N.V. Martemyanova. Nonlocal inverse problem for an equation of elliptic-hyperbolic type. J. Math. Sci., 175(1):39-50, 2011. http://dx.doi.org/10.1007/s10958-011-0331-9.

[16] G.A. Salimova. Inverse boundary value problem for a fourth order partial differential equation with non self-adjoint boundary conditions. In S.K. Ntouyas(Ed.), Proceeding of Instiitute of Mathematics and Mechanics NAS of Azerbaijan, volume XXIX(XXXVII), pp. 167-178, 2008.

[17] A.A. Samarskii. The Theory of Difference Schemes. Marcel Dekker Inc., New York, 2001.

[18] V.V. Solov'ev. Source and coefficient inverse problems for an elliptic equation in a rectangle. Comput.Math. Math. Phys., 47(8):1310-1322, 2007. http://dx.doi.org/10.1134/S096554250708009X. 